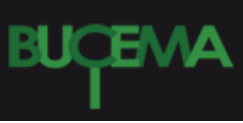

Bulletin du centre d'études médiévales d'Auxerre | BUCEMA

Hors-série $n^{\circ} 5$ | 2013

De Cluny à Auxerre, par la voie des "émotions". Un parcours d'historienne du Moyen Âge : Barbara H. Rosenwein

\title{
Liturgie monastique et société médiévale
}

\section{Alain Rauwel}

\section{(2) OpenEdition \\ Journals}

Édition électronique

URL : https://journals.openedition.org/cem/12534

DOI : $10.4000 /$ cem. 12534

ISSN : 1954-3093

Éditeur

Centre d'études médiévales Saint-Germain d'Auxerre

Référence électronique

Alain Rauwel, «Liturgie monastique et société médiévale », Bulletin du centre d'études médiévales d'Auxerre | BUCEMA [En ligne], Hors-série n ${ }^{\circ} 5$ | 2013, mis en ligne le 29 janvier 2013, consulté le 02 mars 2023. URL : http://journals.openedition.org/cem/12534 ; DOI : https://doi.org/10.4000/cem. 12534

Ce document a été généré automatiquement le 2 mars 2023.

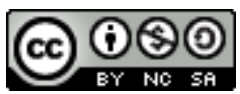

Creative Commons - Attribution - Pas d'Utilisation Commerciale - Partage dans les Mêmes Conditions 4.0 International - CC BY-NC-SA 4.0

https://creativecommons.org/licenses/by-nc-sa/4.0/ 


\title{
Liturgie monastique et société médiévale
}

\author{
Alain Rauwel
}

Entre 1971 et 1991, Barbara Rosenwein a publié trois études aux titres particulièrement significatifs : "Feudal war and monastic peace : Cluniac liturgy as ritual agression» (1971), "Social meaning in the monastic and mendicant spiritualities » (avec Lester Little, 1974), "Monks and their enemies: a comparative approach» (avec sharon Farmer et Thomas Head, 1991). À chaque fois, un terme de caractère nettement religieux (liturgy / spirituality / monks) est associé à un indicateur de structure sociale (agression / social meaning / enemies). Il y a déjà, dans ce choix répété, comme une pétition de principe : ni le rituel ne doit être étudié "hors-monde», ni le social déconnecté du sacral, mais les deux cercles sans cesse en intersection, la connaissance de l'un vivifiant la compréhension de l'autre.

Un tel engagement historiographique ne prend son plein sens, chez B. Rosenwein, que dans un cadre très particulier, qui donne toute sa portée à l'exercice : Cluny. Il ne s'agit pas de céder au discours patrimonialo-touristique qui valorise cluny au nom de sa centralité réelle ou supposée à l'échelle de la Chrétienté médiévale, de son réseau ou de son art. Bien plus profondément, il s'agit de constater que Cluny est, à l'âge féodal, le lieu d'une « liturgisation de la vie entière », universellement présente comme tendance, sans doute, mais jamais portée si près de l'accomplissement ${ }^{1}$. Cela ne se manifeste pas seulement par le quotient considérable du temps éveillé consacré au chœur (que certains spécialistes tendent d'ailleurs aujourd'hui à revoir à la baisse), mais aussi et peut-être surtout par la "ritualisation des activités hors-chœur», circulations, repas, étude, soins du corps... qui amène à dire qu'à Cluny, en somme, tout est liturgie, chaque geste, chaque démarche, aussi banale qu'elle puisse sembler.

3 Nous sommes accoutumés à des perspectives de cet ordre. Il y a quarante ans, elles n'allaient pourtant pas de soi. L'un des grands intérêts d'un retour aux premières études de B. Rosenwein, pour le lecteur de 2012, est de mesurer l'évolution de la bibliographie clunisienne au fil des dernières décennies, avec d'autant plus de curiosité que l'auteur elle-même a abordé la question historiographique dans le premier chapitre 
de Rhinoceros bound. De quoi B. Rosenwein disposait-elle en 1971 pour entrer dans la logique de la vie clunisienne? Suivons les références de bas de pages : en allemand, le grand classique de Sackur, les vues d'ensemble de Dom Schmitz, la thèse discutée de Dom Hallinger. En anglais, les manuels de Dom Butler et de Dom Knowles et les travaux de John Evans. En français, pas grand-chose d'autre que le beau livre, daté, de Guy de Valous. Pour en rester à la question liturgique, il faut reconnaitre que les récentes contributions de Dominique Iogna-Prat ${ }^{2}$, de Catherine Magne sur le sanctoral ${ }^{3}$, de Manuel Ferreira sur la musique ${ }^{4}$, de Ruth Steiner sur les antiennes mariales ${ }^{5}$, pour ne citer que quelques exemples brillants, ont complètement renouvelé notre perception. De même, les recherches sur les coutumes ont connu depuis les dernières années du $\mathrm{xx}^{\mathrm{e}}$ siècle une véritable explosion qui ne permet plus de regarder ces textes, leur nature, leur fonction, avec les mêmes yeux qu'en 1971 -même si le nécessaire travail d'édition n'est pas allé au même rythme ${ }^{6}$. Il n'est pas jusqu'à la communication vraiment fondamentale de Robert Folz sur "Pierre le Vénérable et la liturgie", prononcée l'année suivante ${ }^{7}$, qui demeurât encore inaccessible lorsque B. Rosenwein entra en terrain clunisien.

4 Se trouva-t-elle pour autant seule et désarmée face à l'impressionnant massif de l'office monastique au $\mathrm{XI}^{\mathrm{e}}$ siècle? Un livre était là pour la guider, un livre capital : L'An mil de Georges Duby, paru en 1967. On dira que ce n'était qu'une anthologie - mais c'était une anthologie de Duby! Le choix des extraits, l'exceptionnelle qualité des paragraphes de présentation et de transition, donnent au recueil une grande puissance de suggestion, d'autant plus grande en l'espèce que Duby et Rosenwein se posent au fond la même question : celle de la violence, de sa perception et de sa gestion dans une société qui se veut intégralement chrétienne. Une œuvre comme celle de Raoul le Glabre (Glaber), si abondamment convoquée dans L'An mil, tourne largement autour de ce problème, qui ne peut laisser indifférent aucun historien de la société féodale. Pour Duby lecteur de Glaber, toute la vie du moine roman est un combat contre les forces du mal, avec la prière et la pénitence comme armes à défaut de lance et d'épée. Cela crée une identité structurelle du priant et du combattant, dont les acticités extérieures sont sans doute bien différentes, mais pas la mission fondamentale. B. Rosenwein ne dit pas autre chose en considérant « l'intercession comme arme » et en introduisant, dans le brillant essai de 1974, la notion de « liturgie belliqueuse ».

5 Il y a fort à parier que c'est cette conviction qui a conduit l'historienne à quitter un moment Cluny pour un autre lieu, qui appartient à la préhistoire du monachisme occidental: Saint-Maurice d'Agaune ${ }^{8}$. Agaune est la véritable matrice religieuse et politique de l'espace bourguignon en tant que "modèle monastique des rois burgondes ${ }^{9}$ ». C'est aussi l'une des boucles les plus serrées du nœud gordien de la « liturgie belliqueuse » : en raison de l'identité des saints de la Légion thébaine vénérés là, bien sûr, les saints soldats les plus fameux d'Occident, mais aussi dans la mesure où le système cultuel y reproduit précocement, peut-être dès l'origine ${ }^{10}$, un modèle militaire, celui des turmae, des troupes de moines se relayant pour assurer la laus perennis sur un mode directement emprunté aux légions romaines, par le biais d'autres abbayes rhodaniennes ${ }^{11}$. Des moines soldats du Christ assurant le service de soldats martyrs du Christ : Agaune avait bien vocation à être l'un des centres où fut inventée l'« agressive non-stop liturgy » qu'étudie B. Rosenwein.

6 Il est difficile de mesurer le rôle de l'historienne américaine dans l'acceptation désormais généralisée d'une vision du monachisme féodal fondée non sur la seule 
aspiration à la paix, mais sur une étroite complémentarité entre paix et guerre. Pour les moines-historiens qui vivaient à l'ombre de la devise bénédictine moderne, "Pax", même pour les meilleurs, il était difficile d'admettre que le couple devait être pris dans sa totalité, au-delà de la dévalorisation apparente de l'un de ses termes. C'est un point que Dom Leclercq n'admit jamais vraiment. La lecture des textes monastiques produit pourtant un effet d'évidence : dès la Règle, le vocabulaire militaire est omniprésent ${ }^{12}$. La densité est trop forte pour parler d'une simple évocation, il y a bel et bien assimilation. Les moines de l'an mil, précisément marqués par l'idéologie du retour à la règle ${ }^{13}$, se voulaient des milites Christi au sens le plus plein, menant corps et âme, par l'ascèse, le combat décisif. C'est tout le mérite de Katherine Allen Smith d'avoir pris leurs affirmations au sérieux et, enfin, rassemblé systématiquement des notations jusque là plus effleurées que vraiment analysées ${ }^{14}$. À sa manière, B. Rosenwein l'avait déjà fait pour les aspects liturgiques de la vie claustrale. On peut, à sa suite, dépasser l'analogie pour enregistrer une véritable donnée structurelle.

7 Contre qui était donc dirigée l'agressivité rituelle ? Contre le démon, bien sûr. Mais aussi contre des adversaires très terrestres, ceux que l'on appelait les «ennemis ", reprenant l'un des mots les plus fréquents du psautier, où il est sans cesse question des assauts des inimici. Leur tort est de porter atteinte aux biens ou aux droits des communautés monastiques. Contre eux, la résistance ne peut s'organiser directement, puisque la profession des conseils évangéliques empêche de verser le sang. Pour autant, les procédures alternatives sont nombreuses. On pense évidemment à la clamor, si bien analysée par L. Little ${ }^{15}$. Mais la perspective peut être élargie. Dans « Social meaning in the monastic spirituality », B. Rosenwein souligne que l'action liturgique tout entière est une bataille non sanglante, et pourtant supérieurement efficace. Cette intuition gagne à être prolongée en direction d'une histoire de l'Eucharistie. Qu'est-ce que la messe, en effet, cette messe que les moines du XI ${ }^{\mathrm{e}}$ siècle sont désormais si nombreux à célébrer quotidiennement, sinon la réitération non sanglante de l'unique sacrifice du Calvaire, qui conserve nonobstant une parfaite efficacité? Une telle théologie sacramentaire s'inscrit sans réserve dans le cadre de la "liturgie belliqueuse ». La messe quotidienne des Clunisiens est une bataille contre le mal où le célébrant à l'autel est comme l'avant-garde de la militia christiana que constitue le convent, lui-même armée d'élite de la Respublica christiana dans son ensemble. De ce point de vue, ce n'est certes pas un hasard si les acteurs principaux du grand tournant eucharistique du milieu du $\mathrm{XI}^{\mathrm{e}}$ siècle, où la définition symbolique de filiation augustinienne devient inaudible et se trouve brutalement remplacée par une définition hyper-réaliste, sont des clercs issus du monachisme réformateur : ils étaient les mieux placés pour faire accepter à toute l'Église un système dans lequel l'oblation de l'hostie est la clef du salut.

Reste qu'il y a sans doute une différence notable entre le démon et les inimici des moines : avec le premier, la guerre est destinée à durer jusqu'à la fin des temps, sans trêve; les seconds, en revanche, ne seraient en ligne de mire que pour mieux dramatiser, après la crise provoquée et jouée, leur réintégration dans le monde de la paix. Comme on l'a proposé aussi à propos des affrontements entre grands féodaux, la violence ritualisée des moines s'inscrirait dans un «théâtre de la guerre » visant non pas l'exclusion mais l'inclusion, le retour spectaculaire dans la societas de Cluny. On quitterait donc moins que jamais le domaine de la liturgie, au fil d'une parade d'hostilité orientée vers la réconciliation, comparable, dans une certaine mesure, à ce que décrit le pontifical : la dialectique programmée de l'exclusion des pénitents, au 
début du Carême, dont tout le sens est de préparer leur rentrée dans l'église à la veille de Pâques. B. Rosenwein a noué tous ces fils en une formule magnifique : «the trumpet calls of clamores and penalty clauses were muted by the hidden whispers of societas, socialitas and amicitia ${ }^{16}$ ”.

9 L'amicitia nous conduit tout droit au monde des affects, des émotions, ce "troisième versant » de l'œuvre de B. Rosenwein (après Cluny et les immunités) qui, comme on s'en doutait bien, n'a pas surgi inopinément et travaillait déjà en profondeur les recherches antérieures. En revanche, on sera sensible à l'évolution du discours théorique entre les années 1970 et les années 2000. Au point de départ, la liturgie monastique est décrite comme un refoulement, une domestication de l'agressivité par le rite, où il s'agirait de répondre à l'angoisse née des soubresauts d'une société anomique. Tout cela est très lié au contexte socio-politique américain que décrit ici même L. Little ${ }^{17}$ et dépend encore fortement d'une "psycho-histoire ", très présente aussi en France à ce moment, que précisément l'histoire des émotions, en se formalisant, a cherché à dépasser, non sans succès ${ }^{18}$.

10 Ce qui reste, par-delà les modes théoriques, c'est la question fondamentale du rapport à la violence et de son évolution aux $\mathrm{XI}^{\mathrm{e}}$ et $\mathrm{XII}^{\mathrm{e}}$ siècles. B. Rosenwein a fait usage à ce propos, dans ses premiers travaux, de la notion de "guerre sainte ${ }^{19}$ ". Le sens dans lequel elle l'emploie, parmi toutes les colorations possibles de la formule, est peut-être assez voisin de celui qu'avait retenu Étienne Delaruelle, dont les diverses contributions ne se sont vraiment imposées que lors de leur rassemblement en volume en $1980{ }^{20}$ mais avaient été rédigées dès les années 1960, au croisement d'interrogations portant plutôt sur le pèlerinage et la paix. La forte dimension anthropologique et liturgique de l'acception conserve à cette "guerre sainte » là une validité que d'autres lectures, plus unilatéralement juridiques, ont perdue ${ }^{21}$. Pour autant, la leçon de l'historiographie la plus récente, en cette matière, est une leçon de prudence. Patrick Henriet, à propos du Reconquista und Heiliger Krieg de Bronisch, avait invité à entreprendre un examen systématique de bellum sanctum ${ }^{22}$. Thomas Deswarte a répondu à ce vœu et puissamment éclairci un maquis lexical et idéologique jusque là envahi par les broussailles ${ }^{23}$. Il en ressort que la plupart des bellum sanctum en usage dans nos références viennent non de textes proprement dits mais de titres, donnés tardivement, par des érudits d'Ancien Régime, à des documents acéphales. À l'époque qui nous occupe, on ne rencontrera la formule au sens de "guerre pour Dieu, guerre bénie de Dieu » ni avant ni pendant la première Croisade. Ce n'est qu'après 1100 , lorsque les théologiens et les chroniqueurs tentent d'interpréter les événements orientaux, qu'apparaissent les premières occurrences, chez Guibert de Nogent ou Geroch de Reichersberg; encore ne sont-elles pas bien nombreuses. Il est établi en tout cas qu'auparavant, à la grande époque de la spiritualité clunisienne, bellum sanctum signifie exclusivement combat spirituel contre le démon et les vices - parfaite confirmation, en somme, de l'idée de « liturgie belliqueuse » développée par B. Rosenwein!

11 À la dernière ligne de "Feudal war and monastic peace ", l'historienne américaine note ceci : "men went off to Jerusalem or Cîteaux instead (of Cluny). The day of cluniac liturgy was passed». On pourrait se contenter d'y lire une péroraison classique, bouclant par un «truc» rhétorique («les jours de la liturgie clunisienne étaient passés ») la boucle de la démonstration : on aurait bien tort. Le rapprochement entre Jérusalem et Cîteaux pour caractériser la nouvelle atmosphère spirituelle du XII ${ }^{\mathrm{e}}$ siècle est en effet une très belle et très féconde intuition. L'exactitude voudrait d'ailleurs que 
l'on dise Clairvaux plutôt que Cîteaux, car ce n'est pas à un hypothétique «ordre cistercien" que renvoie le parallèle, mais à la personne même de saint Bernard. Comment ne pas penser, en découvrant la pointe de l'article de B. Rosenwein, au long développement sur la Terre sainte dans le Sermo exhortatorius ad milites Templi ${ }^{24}$ ? On s'est interrogé de longue date sur la portée de cette méditation topographique. Sans doute faut-il répondre, avec Benjamin Demeslay ${ }^{25}$, que la Terre sainte de l'abbé de Clairvaux est cosa mentale. Nulle curiosité pour des paysages ou des traces matérielles chez un Bernard qui s'est bien gardé de franchir la mer! L'espace concret de la vie du Sauveur devient chez lui espace littéraire et rituel (littéraire parce que rituel, rituel parce que littéraire) dans la mesure même où le monde bernardin est tout entier un « espace textuel ». Le Verbe divin réactualisé, on dirait presque « ré-incarné », dans les mots de l'abbé y rassemble une communauté qui en devient elle aussi «communauté textuelle». Dans la pensée de Bernard, Clairvaux comme Jérusalem est une page, la dernière, du livre qu'écrit l'Esprit dans l'histoire. Le monastère du Val d'Absinthe, à ses yeux, vaut bien l'Urbs Jerusalem beata: c'est le terme du vrai pèlerinage, le lieu de la vraie conversion ${ }^{26}$.

Les Clunisiens, qui prêchaient aussi la conversio, puisqu'aussi bien telle est la vraie désignation de la vocation bénédictine, n'auraient jamais songé à tenir de tels propos. Ils ne prétendaient avoir dans leur monastère qu'une autre Rome. L'ambition était peut-être aussi grande, mais d'une portée plus nettement temporelle, dépourvue des chatoiements eschatologiques du discours bernardin. En ce sens, il est légitime de distinguer vers 1100 un dépassement de l'horizon clunisien, non par quelque « déclin de Cluny", plus personne n'oserait avoir recours à un poncif si éculé, mais plus fondamentalement par le caractère inédit de la radicalité cistercienne. Du pèlerinage à une « autre Rome » au transitus vers une «nouvelle Jérusalem », il y a bien eu passage à la limite ; B. Rosenwein nous aide à le penser.

\section{NOTES}

1. Le monachisme comme "ritualisation intégrale de la vie » : telle est aussi la définition de P. LEGENDRE, Leçons VII. Le désir politique de Dieu. Études sur les montagnes de l'État et du Droit, Paris, 1988, p. 347.

2. «La Croix, le moine et l'empereur : dévotion à la Croix et théologie politique à Cluny autour de l'an mil », Haut Moyen Âge: culture, éducation et société (Mélanges P. Riché), Paris, 1990, p. 449-475, repris in Études clunisiennes, Paris, 2002, p. 75-92.

3. Le Sanctoral clunisien, thèse, Paris-I, 2005, résumée in Bucema 10, 2006, p. 265-273 [http:// cem.revues.org/316].

4. Cf. «Liturgie et musique à Cluny ", Cluny ou la puissance des moines, Dossiers d'archéologie $\mathrm{n}^{\circ} 269$, 2002, p. 40-47.

5. Les Antiennes mariales à Cluny et à Lewes, trad. fr. Montbrison, 1991.

6. S. BOYNTON et I. COCHELIN (dir.), From dead of night to end of day: the medieval customs of Cluny, Turnhout, 2005.

7. «Pierre le Vénérable et la liturgie », Pierre Abélard / Pierre le Vénérable, Paris, 1975, p. 143-163. 
8. Cf. «Perennial prayer at Agaune » et « One site, many meanings : Saint-Maurice d'Agaune as a place of power in the Early Middle Ages ».

9. «One site, many meanings » p. 273.

10. B. Rosenwein insiste sur le fait que la première mention de turmae se lit dans une fausse charte de Sigismond, vraisemblablement carolingienne. Peut-être, mais certains des documents qu'elle cite sont indubitablement plus anciens, à commencer par la Vie de sainte Salaberge de Laon, où les nonnes sont décrites "per turmas ad instar Agaunensium », et qui est désormais datée des environs de 680 par M. GAILLARD, « De l'Eigenkloster au monastère royal : l'abbaye SaintJean de Laon du milieu du $\mathrm{VII}^{\mathrm{e}}$ au milieu du $\mathrm{VIII}^{\mathrm{e}}$ s. à travers les sources hagiographiques", L'Hagiographie du Haut Moyen Âge en Gaule du nord: manuscrits, textes et centres de production, Sigmaringen, 2000, p. 249-262 et «Les vitae des saintes Salaberge et Anstrude de Laon, deux sources exceptionnelles pour l'étude de la construction hagiographique et du contexte sociopolitique ", Revue du Nord 93, 2011, p. 655-669 ; sur la richesse historique de la Vita Sadalbergae, A. RAUWEL, «Identité de frontière ? Les confins des diocèses des Lingons, des Leuques et des Séquanes du VII ${ }^{\mathrm{e}}$ au XII ${ }^{\mathrm{e}}$ s. ", Cahiers haut-marnais 248-251, 2007, p. 25-31. Le système des turmae est donc attesté à Agaune $\mathrm{au} \mathrm{VII}{ }^{\mathrm{e}} \mathrm{s}$. J'incline pour ma part à penser qu'il est primitif.

11. Et non par imitation des Acémètes de Constantinople, dont B. Rosenwein a montré dans «Perennial prayer » qu'il était inutile de les convoquer ici. PH. BERNARD a pourtant voulu rétablir l'idée de modèle oriental ( La laus perennis dans la Gaule de l'Antiquité tardive: état des questions et éléments d'un bilan ", Sine musica nulla disciplina : studi in onore di G. Cattin, Padoue, 2006, p. 39-69), suivi par A.-M. HELVETIUS («L'abbaye de Saint-Maurice d'Agaune dans le Haut Moyen Âge ", à paraître dans Autour de saint Maurice, actes du congrès mauricien de 2009). Il convient de maintenir le principe d'une origine gauloise.

12. E. MANNING, "La signification de militare/militia/miles dans la Règle de saint Benoît ", Revue bénédictine 72, 1962, p. 135-138.

13. B. H. ROSENWEIN, « Rules and the Rule at $\mathrm{X}^{\text {th }}$ century Cluny ».

14. K. ALLEN SMITH, War and the making of medieval monastic culture, Woodbridge, 2011. J'exprime toute ma gratitude à E. Magnani, qui m'a fait connaître cet important travail.

15. Benedictine maledictions : liturgical cursing in romanesque France, Ithaca, 1993.

16. "Monks and their enemies" p. 777.

17. Cf. la référence à Gandhi dans «Feudal war and monastic peace ». On ne peut éviter ici de penser à Th. Merton, dont le rôle a été si grand dans la diffusion en Amérique de l'idée et de l'histoire monastiques. À partir du milieu des années 1960, son engagement politique en faveur des droits civiques, contre le colonialisme, la guerre du Vietnam, la bombe, est de plus en plus net : cf. TH. MERTON, Conjectures of a guilty bystander, 1966, ou Faith and violence, 1968. L'écho de ces livres fut immense.

18. Cf. dans ce recueil les contributions de P. Nagy et D. Boquet.

19. «Feudal war and monastic peace » p. 147 : le développement d'une pensée de la « holy war ».

20. L'Idée de croisade au Moyen Âge, Turin, 1980.

21. Par exemple, M. VILley, La Croisade: essai sur la formation d'une théorie juridique, Paris, 1942. S. PIRON, « Congé à Villey », L’Atelier du CRH 1, 2008 [http://acrh.revues.org/314].

22. "L'idéologie de guerre sainte dans le Haut Moyen Âge hispanique », Francia 29, 2002, p. $171-220$.

23. «La guerre sainte en Occident : expression et signification ", Famille, violence et christianisation au Moyen Âge (Mélanges M. Rouche), Paris, 2005, p. 331-349. Je renvoie à cette étude fondamentale pour les preuves textuelles de toutes les affirmations qui suivent.

24. Éd. et trad. Éloge de la nouvelle chevalerie, Paris, 1990 ("Sources chrétiennes », 367). 
25. Bernard de Clairvaux entre texte et monde: un écrivain total au XII siècle ?, Mémoire de Master 1, Université de Poitiers, 2010. Il faut souhaiter la publication, au moins partielle, de ce travail radicalement neuf.

26. Cf. sur ces questions les très belles recherches de M.B. BRUNN, Parables : Bernard of Clairvaux's mapping of spiritual topography, Leiden, 2007 et «Bernard of Clairvaux and the landscape of salvation », A companion to Bernard of Clairvaux, Leiden, 2011, p. 249-278.

\section{AUTEUR}

\section{ALAIN RAUWEL}

Université de Bourgogne, Artehis 